\title{
The Symmetric Longest Queue System
}

\author{
Geert-Jan VAN HOUTUM ${ }^{1}$ \\ Faculty of Mechanical Engineering \\ University of Twente, Enschede, The Netherlands \\ Ivo ADAN and Jan VAN DER WAL \\ Department of Mathematics and Computing Science \\ Eindhoven University of Technology, Eindhoven, The Netherlands
}

\begin{abstract}
We derive the performance of the exponential symmetric longest queue system from two variants: a longest queue system with Threshold Rejection of jobs and one with Threshold Addition of jobs. It is shown that these two systems provide lower and upper bounds for the performance of the longest queue system. Both variants can be analyzed efficiently. Numerical experiments demonstrate the power of the approach.
\end{abstract}

Keywords: Longest queue, Markov chain, performance analysis, bounds.

\section{INTRODUCTION}

In this paper we study the symmetric longest queue system, which is characterized as follows. Consider a system with $N$ types of jobs. Each type has its own queue. In each queue jobs arrive according to a Poisson stream with rate $\lambda$. The jobs are served by one server. The service times are exponentially distributed with mean $1 / \mu$. If the server has completed a job, he picks the next job from the longest queue.

We encountered this model when studying the following problem. A company selling copiers has service contracts with its clients. If a system breaks down

\footnotetext{
${ }^{1}$ Corresponding author. E-mail: g.j.j.a.n.vanhoutum@wb.utwente.nl 
it has to be repaired within 24 hours. Repair usually means replacing one or more parts by spare parts. Often the defected parts can be repaired, think of printed circuit boards. The company has to decide how many spares are needed. Having too many spare parts leads to extra costs as these parts are never used and will become obsolete. On the other hand, having too few spare parts leads to the situation that too often a repair will take more than 24 hours. If there are many different types of spares that have to be repaired and the repair capacity is limited, then it has to be decided which items have to be repaired first. A sensible strategy will be to choose those items which are likely to be needed first. Other examples of the problem described above are found in the repair of medical systems or airplane engines.

Simplifying the problem described above we arrive at the longest queue system. Since the number of copiers is very large (thousands), it is reasonable to assume that defects occur according to homogeneous Poisson processes, one per part-type. Each type has its own queue. All defected parts of similar types are served by a single repairman. If he has completed a job, he has to choose the next job from one of the queues. In this paper we will concentrate on a simplified version of the problem in which all Poisson processes have the same arrival rate and the repair times for all job types are exponentially distributed with the same mean. Because of the symmetry we also assume that the number of spares of each type is the same. Then a natural repair strategy for the repairman is to always select his next job from the longest queue, since the number of good spares of that part type is the smallest. An important performance measure is the fraction of time that all spares are in repair. If during that time a failure in a copier occurs, no good spares are available, so that repair of the copier within 24 hours will be unlikely. So what we need is the probability that there are more than a specified number of defected parts in repair. We will show that this probability can be efficiently obtained.

Note that a realistic model will be far more complicated. The arrival rates and hence the number of spares per type will differ. The jobs will not be exponential and will have distributions that differ per job type. But if one wants to be able to efficiently evaluate realistic models, one should definitely be able to efficiently evaluate the simple model. Also the simple model can be used to obtain some insight in the performance of the longest queue policy.

We will approximate the longest queue model by two other models that are easier to handle and that provide lower and upper bounds for the queue length distribution. These two models exploit the aspect that in the longest queue system high imbalance in the queue lengths is not likely to occur. In the lower and upper bound model the difference in length between the longest and the shortest queue is limited to a prespecified threshold. In the lower bound model this is realized by rejecting an arrival in the longest queue if, due to this arrival, the difference between longest and shortest queue will exceed the threshold. It is intuitively 
obvious that this rejection mechanism leads to shorter queues. If in the upper bound model, due to an arrival in the longest queue, the threshold will be exceeded, a job is added to the (all) shortest queue(s) as well. These extra arrivals clearly lead to longer queues. The larger the threshold, the less jobs will be rejected or added and so the bounds will be better. Since the server acts in a manner trying to balance the queue lengths, one might expect that the bounds will be tight for already moderate values of the threshold.

To prove that these two models indeed produce lower and upper bounds for the queue length distribution of the longest queue system we use a technique similar to the ones used by Van der Wal [9], Van Dijk and Van der Wal [4], Van Dijk and Lamond [3] and Adan et al. [1]. First the Markov processes representing the three models are translated into equivalent Markov chains. Then we show by induction that for each finite number of periods the performance of the longest queue model is sandwiched between the performances of the two threshold models. Letting the number of periods tend to infinity yields the desired result for the average performance. The proofs are presented for the case of two queues only. The proofs for the case of more than two queues are essentially the same, but notationally more complex and therefore omitted.

The exponential longest queue model with two queues has been studied by Zheng and Zipkin [10] who assume that the longest queue policy is applied preemptively. The same model has also been analyzed by Flatto [5] using generating functions. For the model with more than two queues an approximation of the standard deviation of the queue lengths is derived in Zipkin [11]. Cohen [2] presents a generating function analysis of the longest queue model with two queues and generally distributed service times.

The paper is organized as follows. In Section 2 we describe the models and translate the continuous-time Markov processes to discrete-time Markov chains. Section 3 explains the technique used to prove that the performance of one model is better than that of another one. Monotonicity properties of the longest queue model are established in Section 4. In the Sections 5 and 6 it is proved that the threshold models indeed give lower and upper bounds, respectively, for the longest queue model. In Section 7 it is shown how the queue length distributions of the threshold models can be found. Numerical results are presented in Section 8.

\section{THE MODELS}

In the longest queue model we consider $N$ types of jobs. Each type has its own queue. The queues are numbered $1, \ldots, N$. In each queue jobs arrive according to a Poisson stream with rate $\lambda$. The jobs are served by one server. The service times are exponentially distributed with mean $1 / \mu$. If the server has completed a job he picks the next job from the longest queue. Ties are broken with equal probabilities. In the lower and upper bound model the arrival mechanism is modified to accomplish that the difference between the longest and the shortest queue is lim- 
ited to a prespecified threshold $L(\geq 1)$. If, due to an arrival in the longest queue, the difference between the longest and the shortest queue would exceed $L$, then in the lower bound model that job is rejected and in the upper bound model a job is added to all shortest queues as well. The first model will be called Threshold Rejection model, the other one Threshold Addition model.

The state of the original longest queue system will be described by an ordered $N$-tuple of the queue lengths $s=\left(s_{1}, \ldots, s_{N}\right)$ with $s_{1} \leq \ldots \leq s_{N}$. So $s_{1}$ is the length of the shortest queue, $s_{2}$ the length of the second shortest queue, and so on. Because of the symmetry we are not interested in the length of a specific queue. If a job is taken into service, then it is removed from its queue immediately. So in the queues there are waiting jobs only. If all queues are empty there are two possible states $(0, \ldots, 0 ; i)$ and $(0, \ldots, 0 ; b)$ where $i$ stands for an idle server and $b$ for a busy server. The state $(0, \ldots, 0 ; b)$ will be abbreviated as $(0, \ldots, 0)$. Note that this state description does not show which type of job is in service. Thinking of the repair of defected parts in copiers, no essential information is lost by assuming that a part that is in service will still be in time to replace a defected part. Of course, it is easy to include this type number in the state description, but it would further increase the number of states.

The state set in the two bound models is restricted to tuples $s$ with $s_{N}-s_{1} \leq L$.

The three models are Markov processes. In all states the output rate is less than or equal to $N \lambda+\mu$. Without loss of generality we set $N \lambda+\mu=1$. The original longest queue model is ergodic if $N \lambda<\mu$. This easily follows by using that the total number of jobs in the system is stochastically the same as in the $M|M| 1$ system with arrival rate $N \lambda$ and service rate $\mu$. Since the model with Threshold Rejection destroys work, it is ergodic if the original model is ergodic. So the condition $N \lambda<\mu$ is sufficient (but not necessary) for the Threshold Rejection model to be ergodic. In the model with Threshold Addition extra work is created. So there the ergodicity condition will be stronger (see (13) in Section 7). It is, however, intuitively obvious that if the original model is ergodic, then for sufficiently large $L$ the Threshold Addition model will be ergodic as well (a formal proof for $N=2$ is given in [6]). In Figure 1 the transition-rate diagrams for the three models are depicted for $N=2$ and $L=3$. For the threshold models we only depict the differences with respect to the original model. In the Threshold Rejection model the arrival arc with rate $\lambda$ from state $\left(s_{1}, s_{1}+L\right)$ to $\left(s_{1}, s_{1}+L+1\right)$ is redirected to the state itself and in the Threshold Addition model it is redirected to $\left(s_{1}+1, s_{1}+L+1\right)$.

Let $Q$ be the generator of one of the three Markov processes we are dealing with. Then the corresponding equilibrium distribution $p$ satisfies $p Q=0$. Instead of studying the Markov process with generator $Q$ we will consider the Markov chain with transition matrix $P=I+Q$. As $N \lambda+\mu=1$ the matrix $P$ is stochastic. Clearly the equilibrium distributions of the Markov chain and the Markov process 

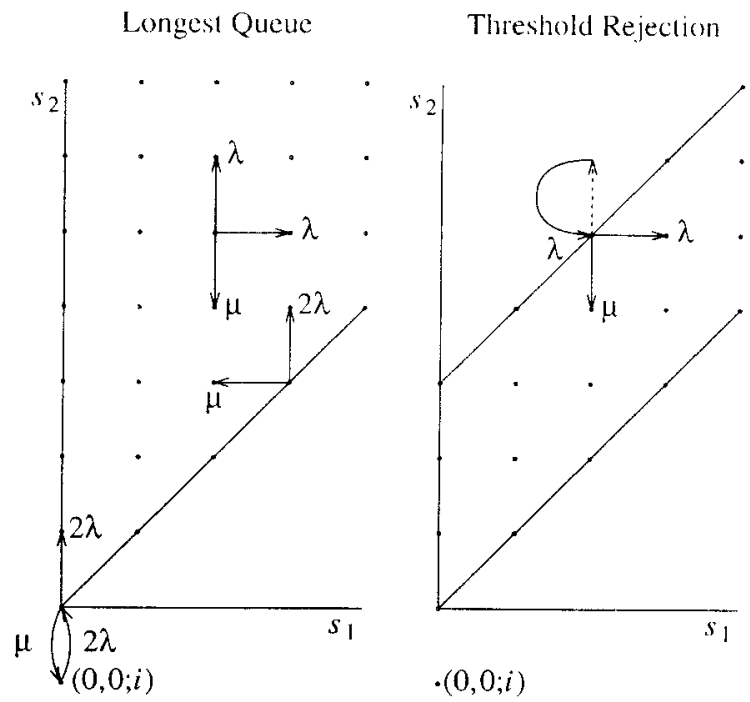

Threshold Addilion

Figure 1. The transition rates for the three models for $N=2$ and $L=3$.

are equal. Also mean costs per unit of time are easy to compare. If $c(s)$ is the cost rate in state $s$ for the Markov process and we take $c(s)$ as the costs per period in the Markov chain, then the Markov process and the Markov chain have the same average costs per unit of time $\Sigma_{s} p(s) c(s)$. From now on we only consider the three Markov chains.

\section{THE LINE OF PROOF}

To prove that the lower and upper bound model indeed give lower and upper bounds for the average cost we study the expected cost over a finite number of periods. Define $v_{n}(s)$ as the expected cost over $n$ periods for the original longest queue model when starting in $s$. Similarly let $u_{n}(s)$ and $w_{n}(s)$ be the expected $n$ period cost for the lower and upper bound model respectively. Defining $u_{0}(s)=v_{0}(s)=w_{0}(s)=0$ for all $s$, we will prove by induction that for all $n$ and all $s$

$$
u_{n}(s) \leq v_{n}(s) \leq w_{n}(s) .
$$

Then it follows that the average costs are ordered in the same way.

An important performance measure is the probability that for a given job type the queue length is equal to or greater than $M$, where $M$ is a fixed nonnegative integer. Therefore we take as cost function $c(s)$ the number of queues with length equal to or longer than $M$. Then the average cost divided by the total number of queues yields the desired probability. 
To prove (1) we will first establish some obvious monotonicity results for the functions $v_{n}$. To keep notations simple we only consider the case $N=2$. One easily sees that the results hold for more than 2 queues as well. The notations, however, become more complex in that case.

\section{MONOTONICITY RESULTS FOR THE FUNCTIONS $v_{n}$}

The monotonicity results that we need are the following intuitively obvious inequalities:

Lemma For all $n \geq 0$ we have

$$
\begin{aligned}
& v_{n}(k, l+1) \geq v_{n}(k, l), \quad 0 \leq k \leq l \\
& v_{n}(k+1, l) \geq v_{n}(k, l), 0 \leq k<l \\
& v_{n}(0,0) \geq v_{n}(0,0 ; i) .
\end{aligned}
$$

The inequalities state that it is preferable to start with smaller queues. Note that the cost function $c(s)$, defined as the number of queues with length at least $M$, also satisfies these inequalities, i.e.,

$$
\begin{aligned}
& c(k, l+1) \geq c(k, l), \quad 0 \leq k \leq l \\
& c(k+1, l) \geq c(k, l), 0 \leq k<l \\
& c(0,0) \geq c(0,0 ; i) .
\end{aligned}
$$

Proof of the lemma: The proof follows by induction. Since $v_{0}=0$ the inequalities trivially hold for $n=0$. Assuming (2)-(4) to hold for $n$ we will establish them for $n+1$.

Proof of (2): We have to distinguish between the three cases $0 \leq k<l, k=l>0$ and $k=l=0$.

Case a: $0 \leq k<l$.

We have

$$
\begin{aligned}
& v_{n+1}(k, l+1)=c(k, l+1)+\lambda v_{n}(k, l+2)+\lambda v_{n}(k+1, l+1)+\mu v_{n}(k, l), \\
& v_{n+1}(k, l)=c(k, l)+\lambda v_{n}(k, l+1)+\lambda v_{n}(k+1, l)+\mu v_{n}(k, l-1) .
\end{aligned}
$$

Comparing the right hand sides of $(8 \mathrm{a})$ and $(8 \mathrm{~b})$ we immediately see that (2) for $n+1$ follows from (5) and the induction assumption for (2)-(4) for $n$.

Case b: $k=l>0$.

Then

$$
\begin{aligned}
& v_{n+1}(k, k+1)=c(k, k+1)+\lambda v_{n}(k, k+2)+\lambda v_{n}(k+1, k+1)+\mu v_{n}(k, k), \\
& v_{n+1}(k, k)=c(k, k)+2 \lambda v_{n}(k, k+1)+\mu v_{n}(k-1, k) .
\end{aligned}
$$


Case c: $k=l=0$.

From

$$
\begin{aligned}
& v_{n+1}(0,1)=c(0,1)+\lambda v_{n}(0,2)+\lambda v_{n}(1,1)+\mu v_{n}(0,0), \\
& v_{n+1}(0,0)=c(0,0)+2 \lambda v_{n}(0,1)+\mu v_{n}(0,0 ; i),
\end{aligned}
$$

we directly get that $v_{n+1}(0,1) \geq v_{n+1}(0,0)$.

Proof of (3): We have to distinguish between $k+1<l$ and $k+1=l$.

Case a: $k+1<l$.

Then

$$
\begin{aligned}
& v_{n+1}(k+1, l)=c(k+1, l)+\lambda v_{n}(k+1, l+1)+\lambda v_{n}(k+2, l)+\mu v_{n}(k+1, l-1), \\
& v_{n+1}(k, l)=c(k, l)+\lambda v_{n}(k, l+1)+\lambda v_{n}(k+1, l)+\mu v_{n}(k, l-1) .
\end{aligned}
$$

So $v_{n+1}(k+1, l) \geq v_{n+1}(k, l)$.

Case b: $k+1=l$, so $(k+1, l)=(l, l)$ and $(k, l)=(l-1, l)$.

We have

$$
\begin{aligned}
& v_{n+1}(l, l)=c(l, l)+2 \lambda v_{n}(l, l+1)+\mu v_{n}(l-1, l), \\
& v_{n+1}(l-1, l)=c(l-1, l)+\lambda v_{n}(l-1, l+1)+\lambda v_{n}(l, l)+\mu v_{n}(l-1, l-1) .
\end{aligned}
$$

Thus $v_{n+1}(l, l) \geq v_{n+1}(l-1, l)$.

Proof of (4): From (9) and (2) and

$$
v_{n+1}(0,0 ; i)=c(0,0 ; i)+2 \lambda v_{n}(0,0)+\mu v_{n}(0,0 ; i),
$$

we immediately see that $v_{n+1}(0,0) \geq v_{n+1}(0,0 ; i)$.

\section{THE LOWER BOUND MODEL; THRESHOLD REJECTION}

Let $L(\geq 1)$ be the threshold. If an arrival in the longest queue leads to a difference of $L+1$ between the queue lengths, then the job is rejected. Define $\delta_{n}:=v_{n}-u_{n}$. Then we show that

$$
\delta_{n}(s) \geq 0
$$

for all $n \geq 0$ and all $s$ that are recurrent in the Threshold Rejection model. The proof follows by induction. For $n=0$ inequality (10) trivially holds. Assuming (10) to hold for $n$ we prove it for $n+1$. We distinguish five cases.

Case a: The states $(k, l)$ with $0 \leq k<l<k+L$.

$$
\delta_{n+1}(k, l)=\lambda \delta_{n}(k, l+1)+\lambda \delta_{n}(k+1, l)+\mu \delta_{n}(k, l-1) \geq 0 .
$$

Case b: The states $(k, k)$ with $k>0$.

$$
\delta_{n+1}(k, k)=2 \lambda \delta_{n}(k, k+1)+\mu \delta_{n}(k-1, k) \geq 0 .
$$

Case c: The states $(k, k+L)$ with $k \geq 0$.

These are the only states in which the outgoing transitions of the original longest queue model and the Threshold Rejection model differ. We have 


$$
\begin{aligned}
u_{n+1}(k, k+L)= & c(k, k+L)+\lambda u_{n}(k, k+L)+\lambda u_{n}(k+1, k+L) \\
& +\mu u_{n}(k, k+L-1) \\
v_{n+1}(k, k+L)= & c(k, k+L)+\lambda v_{n}(k, k+L+1)+\lambda v_{n}(k+1, k+L) \\
& +\mu v_{n}(k, k+L-1)
\end{aligned}
$$

So, using (2),

$$
\begin{aligned}
\delta_{n+1}(k, k+L)= & \lambda v_{n}(k, k+L+1)-\lambda u_{n}(k, k+L)+\lambda \delta_{n}(k+1, k+L) \\
& +\mu \delta_{n}(k, k+L-1) \\
\geq & \lambda v_{n}(k, k+L)-\lambda u_{n}(k, k+L)+\lambda \delta_{n}(k+1, k+L) \\
& +\mu \delta_{n}(k, k+L-1) \\
= & \lambda \delta_{n}(k, k+L)+\lambda \delta_{n}(k+1, k+L)+\mu \delta_{n}(k, k+L-1) \geq 0 .
\end{aligned}
$$

Case d: The state $(0,0)$.

$$
\delta_{n+1}(0,0)=2 \lambda \delta_{n}(1,0)+\mu \delta_{n}(0,0 ; i) \geq 0 .
$$

Case e: The state $(0,0 ; i)$.

$$
\delta_{n+1}(0,0 ; i)=2 \lambda \delta_{n}(0,0)+\mu \delta_{n}(0,0 ; i) \geq 0 .
$$

Conclusion: The Threshold Rejection model gives a lower bound for the average cost in the original longest queue model.

\section{THE UPPER BOUND MODEL; THRESHOLD ADDITION}

Let $L(\geq 1)$ be the threshold. If an arrival in the longest queue leads to a difference of $L+1$ between the queue lengths, then a job is added to the (all) shortest queue(s) as well. The approach is the same as in Section 5. Define $\Delta_{n}:=w_{n}-v_{n}$. We will show that

$$
\Delta_{n}(s) \geq 0
$$

for all $n \geq 0$ and all $s$ that are recurrent in the Threshold Addition model. The proof follows by induction. For $n=0$ inequality (11) trivially holds. Assuming (11) to hold for $n$ we prove it for $n+1$. We have to distinguish the same cases as in Section 5. The only interesting situation is:

Case c: The states $(k, k+L)$ with $k \geq 0$.

$$
\begin{aligned}
w_{n+1}(k, k+L)= & c(k, k+L)+\lambda w_{n}(k+1, k+L+1)+\lambda w_{n}(k+1, k+L) \\
& +\mu w_{n}(k, k+L-1), \\
v_{n+1}(k, k+L)= & c(k, k+L)+\lambda v_{n}(k, k+L+1)+\lambda v_{n}(k+1, k+L) \\
& +\mu v_{n}(k, k+L-1) .
\end{aligned}
$$

So, using (2), 


$$
\begin{aligned}
\Delta_{n+1}(k, k+L)= & \lambda w_{n}(k+1, k+L+1)-\lambda v_{n}(k, k+L+1) \\
& +\lambda \Delta_{n}(k+1, k+L)+\mu \Delta_{n}(k, k+L-1) \\
\geq & \lambda w_{n}(k+1, k+L+1)-\lambda v_{n}(k+1, k+L+1) \\
& +\lambda \Delta_{n}(k+1, k+L)+\mu \Delta_{n}(k, k+L-1) \\
= & \lambda \Delta_{n}(k+1, k+L+1)+\lambda \Delta_{n}(k+1, k+L)+\mu \Delta_{n}(k, k+L-1) \geq 0 .
\end{aligned}
$$

Conclusion: The Threshold Addition model gives an upper bound for the average cost in the original longest queue model.

Remark 1 From the proof in the Sections 4-6 it is clear that the ordering (1) holds for any cost function $c(s)$ satisfying the properties (5)-(7). In particular, this implies for $c(s)$ defined as the number of queues with length at least $M$, that the ordering (1) holds for all $M \geq 0$. Hence we can conclude that for a given job type the queue lengths are ordered stochastically, and thus also the moments of the queue length are ordered.

Remark 2 The same technique can be used to show that the average cost in the lower (upper) bound model increases (decreases) as $L$ increases. The monotonicity can be proved by comparing the models with threshold $L$ and $L+1$. We then need the properties (2)-(4) for the model with threshold $L+1$. They can be proved in a similar way as in Section 4.

\section{ANALYSIS OF THE BOUND MODELS}

From here on we consider the case with $N(\geq 2)$ queues again. We only present the analysis of the model with Threshold Addition. The one of the model with Threshold Rejection is almost identical (the question of ergodicity is easier, see Section 2). The analysis is based on the matrix-geometric theory developed by Neuts [7].

The Threshold Addition model can be described as an irreducible Markov chain with a state space consisting of the $N$-tuples $s=\left(s_{1}, \ldots, s_{N}\right)$ with $s_{1} \leq \ldots \leq s_{N} \leq s_{1}+L$. So $s_{1}$ is the length of the shortest queue, $s_{2}$ is the length of the second shortest queue, and so on and the difference between the longest and the shortest queue is at most $L$. The state space can be partitioned into the single state $(0, \ldots, 0 ; i)$ and the levels $0,1, \ldots$, where level $l$ is defined as the set of states $s$ with $s_{1}=l$. The states at a level are ordered lexicographically. For this partitioning the transition matrix $P$ is of the form

$$
P=\left(\begin{array}{cccccc}
B_{00} & B_{01} & 0 & 0 & 0 & \ldots \\
B_{10} & A_{1} & A_{0} & 0 & 0 & \ldots \\
0 & A_{2} & A_{1} & A_{0} & 0 & \ldots \\
0 & 0 & A_{2} & A_{1} & A_{0} & \ldots \\
\vdots & \vdots & \vdots & \ddots & \ddots & \ddots
\end{array}\right]
$$


in which $A_{0}, A_{1}$ and $A_{2}$ are $m \times m$ matrices and $B_{00}, B_{01}$ and $B_{10}$ are $1 \times 1,1 \times m$ and $m \times 1$ matrices respectively, where $m$ is the number of states at a level, so

$$
m=\left(\begin{array}{c}
N+L-1 \\
L
\end{array}\right) \text {. }
$$

Since two states at levels $>0$ can always reach each other via paths not passing through level 0 , it follows that the stochastic matrix $A_{0}+A_{1}+A_{2}$ is irreducible. Hence we can apply Theorem 1.3.2 in [7] stating that the Markov chain $P$ is ergodic if and only if

$$
\pi A_{0} e<\pi A_{2} e
$$

where $e$ is the column vector of ones and $\pi$ is the unique solution of

$$
\pi=\pi\left(A_{0}+A_{1}+A_{2}\right), \quad \pi e=1 .
$$

Let us assume that condition (13) is satisfied. Then the equilibrium probability vector $p$ exists. By partitioning the vector $p$ into $p(0, \ldots, 0 ; i)$ and into the sequence of vectors $p_{0}, p_{1}, \ldots$, where $p_{l}$ is the equilibrium probability vector of level $l$, we will show that

$$
p_{l}=p_{0} R^{l}, \quad l \geq 0,
$$

for some nonnegative matrix $R$. Of course, this is well-known from the theory in [7], where $R$ is characterized as the minimal nonnegative solution of a quadratic matrix equation. But in our case the special structure of $A_{2}$ can be exploited to directly (without using results from [7]) obtain the matrix $R$ in closed form (see also Ramaswami and Latouche [8]). Since it is only possible to jump from level $l(\geq 1)$ to level $l-1$ via state $(l, \ldots, l)$ to state $(l-1, l, \ldots, l)$ with probability $\mu$, it follows that $A_{2}$ has only one nonzero entry, namely $\mu$ in the first row and $k$ th column, where $k$ denotes the position of $(l-1, l, \ldots, l)$ in level $l-1$. So $A_{2}$ can be written as

$$
A_{2}=\mu e_{1} e_{k}^{T}
$$

where $e_{i}$ is the $i$ th unity column vector. Balancing the flow between level $l(\geq 0)$ and level $l+1$ yields

$$
p_{l} A_{0} e=p_{l+1} A_{2} e=p_{l+1} \mu e_{1} e_{k}^{T} e=p_{l+1} \mu e_{1} .
$$

Hence

$$
p_{l+1} A_{2}=p_{l+1} \mu e_{1} e_{k}^{T}=p_{l} A_{0} e e_{k}^{T} .
$$

Substitution of this relation into the equilibrium equations at level $l(\geq 1)$, i.e. into

$$
p_{l}=p_{l-1} A_{0}+p_{l} A_{1}+p_{l+1} A_{2},
$$

leads to

$$
p_{l}=p_{l-1} R,
$$

where 


$$
R=A_{0}\left(I-A_{1}-A_{0} e e_{k}^{T}\right)^{-1},
$$

and $I$ is the $m \times m$ identity matrix. The inverse of $I-A_{1}-A_{0} e e_{k}^{T}$ exists (and is nonnegative), since it is a substochastic matrix. The representation of $R$ concurs with expression (10) in [8]. From (15) the desired result (14) easily follows.

To finally complete the solution (14) the probability $p(0, \ldots, 0 ; i)$ and the vector $p_{0}$ have to be solved from the boundary conditions

$$
\begin{aligned}
& p(0, \ldots, 0 ; i)=p(0, \ldots, 0 ; i) B_{00}+p_{0} B_{10}, \\
& p_{0}=p(0, \ldots, 0 ; i) B_{01}+p_{0}\left(A_{1}+R A_{2}\right),
\end{aligned}
$$

together with the normalization equation

$$
p(0, \ldots, 0 ; i)+\sum_{l=0}^{\infty} p_{l} e=1 .
$$

By substituting the form (14) for $p_{l}$, the sum of all $p_{l} e$ can be rewritten as

$$
\sum_{l=0}^{\infty} p_{l} e=p_{0} \sum_{l=0}^{\infty} R^{l} e=p_{0}(I-R)^{-1} e .
$$

The convergence of the series of powers $R^{l}$ follows from the finiteness of the sum of all $p_{l} e$ (the Markov chain $P$ is ergodic) and the fact that all components of $p_{0}$ are positive (the Markov chain $P$ is irreducible). Hence, equation (16) simplifies to

$$
p(0, \ldots, 0 ; i)+p_{0}(I-R)^{-1} e=1 .
$$

\section{NUMERICAL RESULTS}

Let the random variable $K_{i}$ denote the number of type $i$ jobs waiting for service. We have shown in the previous sections that we can compute lower and upper bounds for the probability $P\left(K_{i} \geq M\right)$. Note that by symmetry $K_{1}, \ldots, K_{N}$ all have the same distribution. Thinking of the repair of copiers, then an important performance measure is the service level $\beta(M)$ defined as the fraction of defects that can be repaired in time given that we have $M$ spares of each part type. To determine $\beta(M)$ we need the probability $P\left(K_{i} \geq M\right)$. Namely, if at the time a defect occurs $M$ or more parts of the required type are in repair, then no good spare is immediately available, so the defected part cannot be replaced by a good spare in time. Hence, by using the PASTA property of Poisson arrivals, it follows that

$$
\beta(M)=1-P\left(K_{i} \geq M\right)=1-\frac{1}{N} \sum_{s} c(s) p(s),
$$

where as before $c(s)$ is defined as the number of queues with length at least $M$.

What we want to know is the minimal $M$ such that a given target service level $\beta$ is satisfied. The two threshold models provide a lower and upper bound for the minimal $M$. By subsequently computing the lower and upper bound for $L=1$, $L=2$, and so on, until the lower and upper bound coincide, the minimal $M$ can be 
determined exactly. In Figure 2 we demonstrate the rate of convergence of the lower and upper bounds for $\beta(M)$ for the case $N=4$ and workload $\rho=0.9$. The workload is defined by $\rho=N \lambda / \mu$. We used a logarithmic axis for $\beta(M)$ to blow up the relevant region near 1 . For the Threshold Addition model we can not show results for $L=1$ and $L=2$, since for these thresholds the model is not ergodic. The results for the longest queue model (solid line) are obtained from the two threshold models with $L=8$. The example shows that the bounds rapidly converge (we conjecture that the convergence is exponentially fast, see also [6]). Note that the lines in Figure 2 are nearly straight. This implies that the probabilities $P\left(K_{i}=M\right)$ behave geometrically for already small values of $M$.

In Table 1 we list for increasing values of $N$ and $\rho$ the minimal $M$ needed to satisfy the target service level $\beta$ which is varied as $0.9,0.95$ and $0.99 . L$ denotes the minimal threshold for which the lower and upper bound for $M$ coincide and $m$ is the number of states at a level for that value of $L$ (see (12)). The amount of work needed to solve the threshold models is proportional to $\mathrm{m}^{3}$. (In the two examples marked with $*$ the target service level may not be satisfied. There the threshold models guarantee that $0.8999 \leq \beta(6) \leq 0.9004$ for $N=8$ and $\rho=0.95$, and $0.9499 \leq \beta(2) \leq 0.9501$ for $N=10$ and $\rho=0.8$.)

From the results in Table 1 we see that $M$ can be determined exactly for already small values of the threshold $L$, even for high values of the workload $\rho$, and that the thresholds are smaller for larger systems.

We will now compare the performance of this longest queue (LQ) policy with that of the first-come first-served (FCFS) policy. To derive the service level $\beta(M)$ for the FCFS policy we first introduce the random variable $K_{T O T}$ denoting the total number of jobs waiting for service. Note that

$$
P\left(K_{T O T}=n\right)= \begin{cases}(1-\rho)(1+\rho), & n=0, \\ (1-\rho) \rho^{n+1}, & n>0 .\end{cases}
$$

It follows that

$$
P\left(K_{i} \geq M\right)=\sum_{l=M}^{\infty} P\left(K_{i}=l\right)=\sum_{l=M}^{\infty} \sum_{n=l}^{\infty} P\left(K_{i}=l \mid K_{T O T}=n\right) P\left(K_{T O T}=n\right) .
$$

Using that $K_{i} \mid K_{T O T}=n$ is binomially distributed with success probability $1 / N$ and $n$ trials, we find after some algebra

$$
P\left(K_{i} \geq M\right)=\frac{\rho^{M+1}}{(N-(N-1) \rho)^{M}} .
$$

Hence, for the FCFS policy we obtain

$$
\beta(M)=1-P\left(K_{i} \geq M\right)=1-\frac{\rho^{M+1}}{(N-(N-1) \rho)^{M}} .
$$

Of course, for each part type the mean number of parts in repair is the same for both policies. But we expect that under the LQ policy the variance of this number 


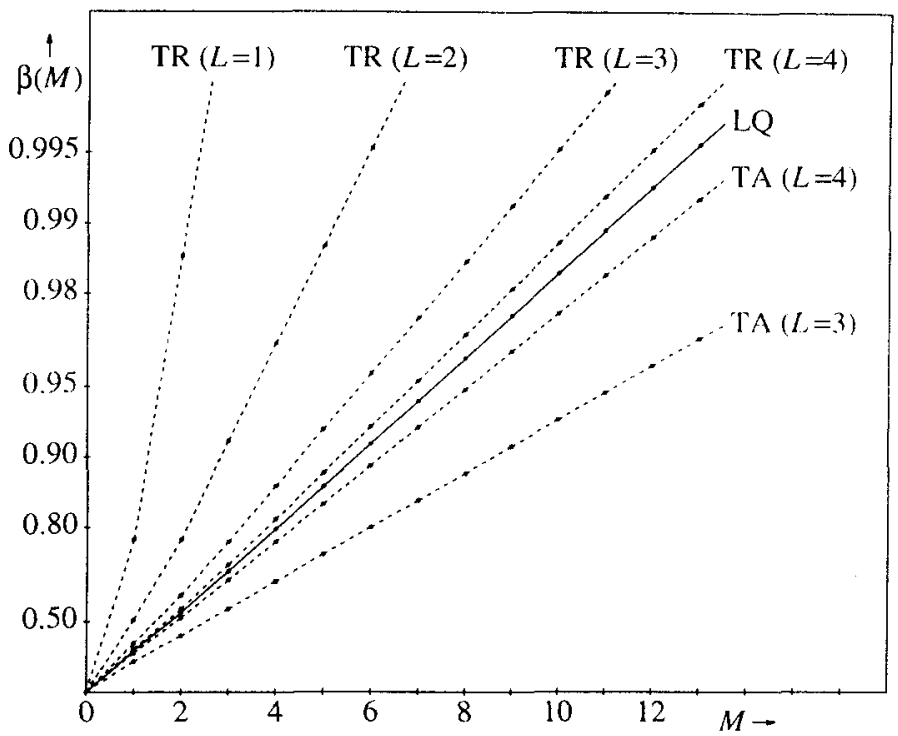

Figure 2. Service levels for longest queue ( $L Q)$, Threshold Rejection (TR) and Threshold Addition (TA) for $N=4$ and $\rho=0.9$.

Table 1. The minimal number of spares needed for each part type to satisfy the target service level.

\begin{tabular}{|c|c|c|c|c|c|c|c|c|c|c|c|c|}
\hline \multirow{2}{*}{$\beta$} & & \multicolumn{3}{|c|}{$N=2$} & $N=4$ & $N=6$ & \multicolumn{3}{|c|}{$N=8$} & \multicolumn{3}{|c|}{$N=10$} \\
\hline & & $M$ & $L$ & $m$ & $M L m$ & $M L \quad m$ & $M$ & $L$ & $m$ & $M$ & $L$ & $m$ \\
\hline \multirow[t]{4}{*}{0.90} & 0.50 & 2 & 2 & 3 & 2320 & 1221 & 1 & 2 & 36 & 1 & 2 & 55 \\
\hline & 0.80 & 6 & 7 & 8 & 3435 & $\begin{array}{lll}33 & 56\end{array}$ & 2 & 4 & 330 & 2 & 3 & 220 \\
\hline & 0.90 & 11 & 6 & 7 & 6556 & $\begin{array}{lll}5 & 6 & 462\end{array}$ & 4 & 4 & 330 & 3 & 4 & 715 \\
\hline & 0.95 & 23 & 7 & 8 & 12556 & 85252 & $6^{*}$ & 6 & 1716 & 5 & 5 & 2002 \\
\hline \multirow[t]{4}{*}{0.95} & 0.50 & 3 & 3 & 4 & 2210 & $\begin{array}{lll}22 & 21\end{array}$ & 2 & 2 & 36 & 1 & 3 & 220 \\
\hline & 0.80 & 7 & 4 & 5 & 4435 & $\begin{array}{lll}34 & 126\end{array}$ & 3 & 3 & 120 & $2^{*}$ & 5 & 2002 \\
\hline & 0.90 & 15 & 8 & 9 & 8556 & 65252 & 5 & 5 & 792 & 4 & 4 & 715 \\
\hline & 0.95 & 29 & 10 & 11 & 15684 & 116462 & 8 & 5 & 792 & 7 & 4 & 715 \\
\hline \multirow[t]{4}{*}{0.99} & 0.50 & 4 & 3 & 4 & 3210 & 2356 & 2 & 2 & 36 & 2 & 2 & 55 \\
\hline & 0.80 & 11 & 5 & 6 & 6435 & 55252 & 4 & 3 & 120 & 3 & 4 & 715 \\
\hline & 0.90 & 22 & 6 & 7 & 12684 & 85252 & 6 & 5 & 792 & 5 & 5 & 2002 \\
\hline & 0.95 & 45 & 8 & 9 & 23684 & 165252 & 12 & 5 & 792 & 10 & 5 & 2002 \\
\hline
\end{tabular}




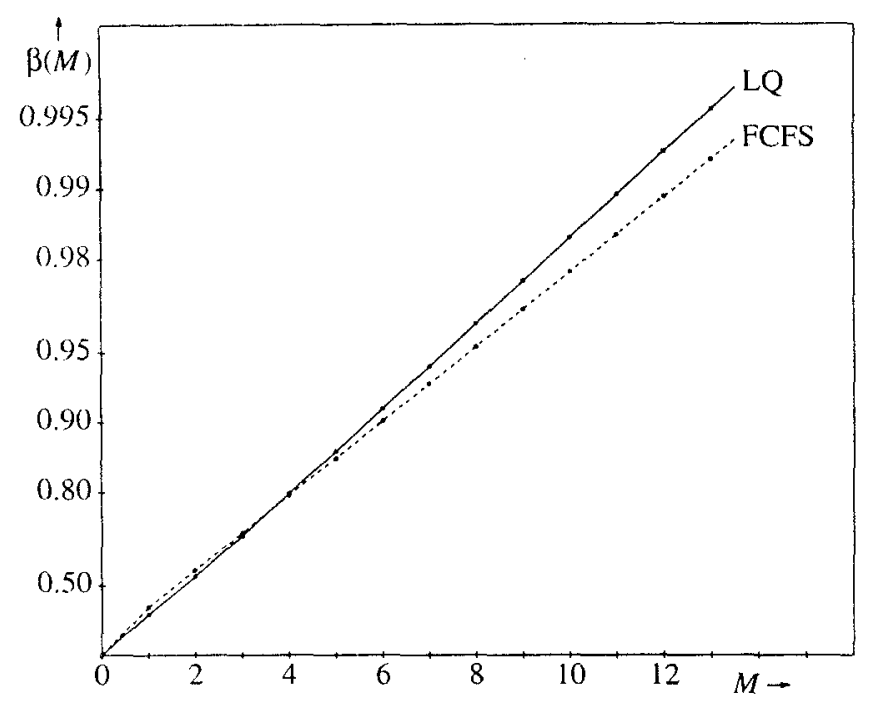

Figure 3. Service levels for the $L Q$ and $F C F S$ policy for $N=4$ and $\rho=0.9$.

is smaller than under FCFS. In fact, this has been proved for two queues in [10]. As a consequence, for high service levels less spares may be needed under LQ than under FCFS. This is exactly what can be seen in Figure 3, where for $N=4$ and $\rho=0.9$ the service levels for both policies are displayed (again a logarithmic axis is used for $\beta(M)$ ). Figure 3 also shows that for low service levels FCFS may be a little better than LQ.

In Table 2 we list for several values of $N, \rho$ and $\beta$ the minimal number of spares needed of each part type to satisfy the target service level $\beta$ under the LQ policy. The numbers in parentheses denote the extra spares needed for each part type under the FCFS policy. We see that when the workload is high LQ is in many cases somewhat better than FCFS. Only in two cases (with low workload) FCFS is a little better than LQ. Overall, we may conclude that the differences between LQ and FCFS are small. Observe that for LQ and fixed $\rho$ and $\beta$ the total number of spares $N M$ appears to be fairly constant, provided of course $N$ is not too large.

\section{CONCLUDING REMARKS}

We have seen that it is possible to derive tight bounds for the queue length probabilities in the exponential longest queue system by comparing it with the longest queue system with Threshold Addition and the one with Threshold Rejection. The two threshold systems have an explicit matrix-geometric solution, and therefore, they are much easier to handle than the original system. 
Table 2. Comparison of the performance of the $L Q$ and FCFS policy.

\begin{tabular}{|rc||rrrrrrrrrr|}
\hline$\beta$ & $\rho$ & $N=1$ & $N=2$ & $N=3$ & $N=4$ & $N=5$ & $N=6$ & $N=7$ & $N=8$ & $N=9$ & $N=10$ \\
\hline \hline 0.90 & 0.40 & $2(0)$ & $2(-1)$ & $1(0)$ & $1(0)$ & $1(0)$ & $1(0)$ & $1(0)$ & $1(0)$ & $1(0)$ & $1(0)$ \\
0.60 & $4(0)$ & $3(0)$ & $2(0)$ & $2(0)$ & $2(0)$ & $2(0)$ & $2(0)$ & $2(-1)$ & $1(0)$ & $1(0)$ \\
& 0.80 & $10(0)$ & $6(0)$ & $4(0)$ & $3(0)$ & $3(0)$ & $3(0)$ & $2(1)$ & $2(0)$ & $2(0)$ & $2(0)$ \\
& 0.90 & $21(0)$ & $11(0)$ & $8(1)$ & $6(0)$ & $5(0)$ & $5(0)$ & $4(0)$ & $4(0)$ & $3(1)$ & $3(0)$ \\
& 0.95 & $44(0)$ & $23(0)$ & $15(1)$ & $12(0)$ & $10(0)$ & $8(1)$ & $7(1)$ & $6(1)$ & $6(0)$ & $5(1)$ \\
\hline 0.95 & 0.40 & $3(0)$ & $2(0)$ & $2(0)$ & $2(0)$ & $1(0)$ & $1(0)$ & $1(0)$ & $1(0)$ & $1(0)$ & $1(0)$ \\
0.60 & $5(0)$ & $3(0)$ & $3(0)$ & $2(0)$ & $2(0)$ & $2(0)$ & $2(0)$ & $2(0)$ & $2(0)$ & $2(0)$ \\
0.80 & $13(0)$ & $7(0)$ & $5(0)$ & $4(0)$ & $4(0)$ & $3(1)$ & $3(0)$ & $3(0)$ & $3(0)$ & $2(1)$ \\
0.90 & $28(0)$ & $15(0)$ & $10(1)$ & $8(0)$ & $7(0)$ & $6(0)$ & $5(1)$ & $5(0)$ & $4(1)$ & $4(0)$ \\
0.95 & $58(0)$ & $29(1)$ & $20(1)$ & $15(1)$ & $12(1)$ & $11(0)$ & $9(1)$ & $8(1)$ & $7(1)$ & $7(0)$ \\
\hline 0.99 & 0.40 & $5(0)$ & $3(0)$ & $3(0)$ & $2(0)$ & $2(0)$ & $2(0)$ & $2(0)$ & $2(0)$ & $2(0)$ & $2(0)$ \\
0.60 & $9(0)$ & $5(0)$ & $4(0)$ & $3(1)$ & $3(0)$ & $3(0)$ & $3(0)$ & $3(0)$ & $2(1)$ & $2(1)$ \\
0.80 & $20(0)$ & $11(0)$ & $8(0)$ & $6(1)$ & $5(1)$ & $5(0)$ & $4(1)$ & $4(0)$ & $4(0)$ & $3(0)$ \\
0.90 & $43(0)$ & $22(1)$ & $15(1)$ & $12(1)$ & $10(1)$ & $8(1)$ & $7(1)$ & $6(2)$ & $6(1)$ & $5(2)$ \\
0.95 & $89(0)$ & $45(1)$ & $30(2)$ & $23(1)$ & $19(1)$ & $16(1)$ & $14(1)$ & $12(1)$ & $11(1)$ & $10(1)$ \\
\hline
\end{tabular}

It is also possible to derive bounds for the performance of the (a)symmetric longest system with phase-type interarrival and service times. In this case, however, the state space is much larger than in the exponential case, since we have to include information of the arrival and service process in the state description. Therefore we will only be able to compute the performance of non-exponential systems with a sufficiently small number of queues.

Numerical experiments suggest that the queue length probabilities in the exponential longest queue system behave geometrically for already a small number of jobs in the queue. This property may be exploited to develop simple and good approximations.

Finally, comparison with FCFS showed that there is not much difference between the performance of FCFS and LQ.

\section{REFERENCES}

1. ADAN, I.J.B.F., HOUTUM, G.J. VAN, AND WAL, J. VAN DER, "'Upper and lower bounds for the waiting time in the symmetric shortest queue system," Annals of Operat. Research, vol. 48, pp. 197-217, 1994.

2. COHEN, J.W., "A two-queue, one-server model with priority for the longer queue," Queueing systems, vol. 2, pp. 261-283, 1987. 
3. DIJK, N.M. VAN AND LAMOND, B.F., "Simple bounds for finite singleserver exponential tandem queues," Opns. Res., vol. 36, pp. 470-477, 1988.

4. DIJK, N.M. VAN AND WAL, J. VAN DER, "Simple bounds and monotonicity results for finite multi-server exponential tandem queues," Queueing Systems, vol. 4, pp. 1-16, 1989.

5. Flatto, L., "The longer queue model," Prob. Engineer. Inform. Sci., vol. 3, pp. 537-559, 1989.

6. HAVIV, M. AND HOUTUM, G.J. VAN, "The critical offered load in variants of the symmetric shortest and longest queue systems," LPOM-95-13, University of Twente, Faculty of Mechanical Engineering, 1995.

7. NEUTS, M.F., Matrix-geometric solutions in stochastic models, Johns Hopkins University Press, Baltimore, 1981.

8. RAMASWAMI, V. AND LATOUCHE, G., "A general class of Markov processes with explicit matrix-geometric solutions," OR Spektrum, vol. 8, pp. 209-218, 1986.

9. WAL, J. VAN DER, "Monotonicity of the throughput of a closed exponential queueing network in the number of jobs,' OR Spektrum, vol. 11, pp. 97-100, 1989.

10. ZHENG, Y.S. AND ZIPKIN, P., "A queuing model to analyze the value of centralized inventory information," Opns. Res., vol. 38, pp. 296-307, 1990.

11. ZIPKIN, P., "Performance analysis of a multi-item production-inventory system under alternative policies,'” Mgmt. Sci., vol. 41, pp. 690-703, 1995.

$\begin{array}{lc}\text { Received: } & 11 / 9 / 1994 \\ \text { Revised: } & 2 / 20 / 1996 \\ \text { Accepted: } & 5 / 20 / 1996\end{array}$

Recommended by Hans Daduna, Editor 